PROCEEDINGS OF THE

AMERICAN MATHEMATICAL SOCIETY

Volume 140, Number 1, January 2012, Pages 65-68

S 0002-9939(2011)10982-1

Article electronically published on May 10, 2011

\title{
LARGE SUBGROUPS OF A FINITE GROUP OF EVEN ORDER
}

\author{
BERNHARD AMBERG AND LEV KAZARIN
}

(Communicated by Jonathan I. Hall)

\begin{abstract}
It is shown that if $G$ is a group of even order with trivial center such that $|G|>2\left|C_{G}(t)\right|^{3}$ for some involution $t \in G$, then there exists a proper subgroup $H$ of $G$ such that $|G|<|H|^{2}$. If $|G|>\left|C_{G}(t)\right|^{3}$ and $k(G)$ is the class number of $G$, then $|G| \leq k(G)^{3}$.
\end{abstract}

\section{INTRODUCTION}

Let $G$ be a group of even order and $t$ an involution of $G$. R. Brauer and K.A. Fowler proved in [1] that if $G$ is a finite simple group of even order, then $|G|<\left(\left|C_{G}(t)\right|^{2}\right)$ !; i.e. there are only finitely many finite simple groups with a given centralizer of an involution. It is well-known that if $G$ has at least two non-conjugate involutions, then the order of $G$ is strictly less than $\left|C_{G}(t)\right|^{3}$ for some involutio'n $t$ of $G$. Using the classification of the finite simple groups, H. Yamaki proved in 7 ] that this also holds in general, even if $G$ has only one class of involutions. Moreover, also using the classification, A. Lev has proved in 5. that every finite group $G$ has a subgroup $H$ such that $|H|>|G|^{1 / 2}$.

Recently K. Harada and M. Miyamoto in [3] obtained the following bound, the proof of which does not depend on the classification.

Let $G$ be a finite group with a single class of involutions. For $g \in G$ the extended centralizer $C_{G}^{*}(g)$ of $G$ is defined by $C_{G}^{*}(g)=\left\{x \in G \mid g^{x}=g^{ \pm 1}\right\}$. Let $\Omega_{\pi}$ be the set of all $\pi$-elements of $G$, where $\pi$ is a set of odd primes satisfying a certain restriction connected with the structure of the Grünberg-Kegel graph of $G$, which we omit here (see [3]).

Let $t$ be an involution of $G$ and $H=C_{G}(t)$. If the Sylow 2-subgroups of $G$ are non-cyclic and not (generalized) quaternion, then the following holds:

$$
|G|<|H|^{3}+m_{\pi}|H|^{2}
$$

where $m_{\pi}=\max \left\{|H|,\left|t^{G} \cap C_{G}^{*}(g)\right| \mid 1 \neq g \in \Omega_{\pi}\right\}$. In particular, if $|H|=m_{\pi}$, then $|G|<2\left|C_{G}(t)\right|^{3}$.

In the following we will show that a similar result, not depending on the classification of the finite simple groups, can be obtained using the following result due to E.P. Wigner [6], Theorem 2 (see also [2]).

Received by the editors November 3, 2010.

2010 Mathematics Subject Classification. Primary 20D05, 20 D06.

Key words and phrases. Group, subgroup, centralizer, involution, conjugacy class, square root.

The second author is grateful to the Department of Mathematics of the University of Mainz for its warm hospitality during the time when this reseach was done. He would also like to thank the Deutsche Forschungsgemeinschaft for its financial support. 
Let $G$ be a finite group, and for $g \in G$ let $\zeta(g)$ be the number of the square roots of $g$ in $G$, i.e. the cardinality of the set of the elements $x \in G$ such that $x^{2}=g$. Then Wigner's inequality is as follows:

$$
\sum_{g \in G}\left|C_{G}(g)\right|^{2} \geq \sum_{g \in G} \zeta(g)^{3} .
$$

Using this we will prove the following.

Theorem 1.1. Let $G$ be a finite group of even order with $O_{2}(Z(G))=1$ and let $t$ be an involution in $G$. Then either $|G|<2\left|C_{G}(t)\right|^{3}$ or there exists a proper subgroup $H$ of $G$ such that $|G| \leq|H|^{2}$.

Another result of this type is the following:

Theorem 1.2. Let $G$ be a finite group of even order and let $t$ be an involution in $G$. If $|G|>2\left|C_{G}(t)\right|^{3}$, then there exists an element $1 \neq x \in G$ such that $\left|G: C_{G}(x)\right| \leq k(G)-1$, where $k(G)$ is the class number of $G$. Moreover, either $|G|<\left|C_{G}(t)\right|^{3}$ or $|G| \leq k(G)^{3}$.

\section{Proofs}

Proof of Theorem 1.1. Clearly $\zeta(1)$ is the number of elements of order 2 or 1 , and so $\left|G: C_{G}(t)\right|<\zeta(1)$. Hence, by Wigner's inequality, we have

$$
\sum_{g \in G}\left|C_{G}(g)\right|^{2}>\frac{|G|^{3}}{\left|C_{G}(t)\right|^{3}}
$$

The left side of (2) can be rewritten as $\sum_{i=1}^{k}|G|\left|C_{G}\left(y_{i}\right)\right|$, where $k=k(G)$ and the $y_{i}$ are the representatives of the conjugacy classes of $G, y_{k}=1$.

Suppose first that the center of $G$ is trivial. Then we have

$$
(k-1)|G|\left|C_{G}\left(x_{0}\right)\right|+|G|^{2}>\frac{|G|^{3}}{\left|C_{G}(t)\right|^{3}},
$$

where $\left|C_{G}\left(x_{0}\right)\right|=\max _{i=1}^{k-1}\left|C_{G}\left(y_{i}\right)\right|$. Dividing both sides by $|G|$, we obtain

$$
(k-1)\left|C_{G}\left(x_{0}\right)\right|>|G|\left(\frac{|G|}{\left|C_{G}(t)\right|^{3}}-1\right) .
$$

If $|G|>2\left|C_{G}(t)\right|^{3}$, then $(k-1)\left|C_{G}\left(x_{0}\right)\right|>|G|$. Hence

$$
\left|G: C_{G}\left(x_{0}\right)\right|<(k-1) .
$$

Using the class equation, we have $\left(y_{k}=1\right)$

$$
\sum_{i=1}^{k} 1 /\left|C_{G}\left(y_{i}\right)\right|=1=\sum_{i=1}^{k-1} 1 /\left|C_{G}\left(y_{i}\right)\right|+1 /|G| .
$$

This implies that $(k-1) /\left|C_{G}\left(x_{0}\right)\right|+1 /|G| \leq 1$ and thus $\left|C_{G}\left(x_{0}\right)\right|>k-1$. By (5) this gives the following bound:

$$
|G|<(k-1)\left|C_{G}\left(x_{0}\right)\right|<\left|C_{G}\left(x_{0}\right)\right|^{2} .
$$

Thus in the case $Z(G)=1$ Theorem 1.1 is proved.

Let $K$ be the hypercenter of $G$. Since $O_{2}(Z(G))=1$, then $K$ is of odd order. Denote by $\bar{G}$ the group $G / K$ and use the standard bar convention. If $|\bar{G}| \leq 2\left|C_{\bar{G}}(\bar{t})\right|^{3}$, then $\left|C_{G}(t)\right|=|K|\left|C_{\bar{G}}(\bar{t})\right|$ and $|G|=|K||\bar{G}|<2|K|^{3}\left|C_{\bar{G}}(\bar{t})\right|^{3}$, and there is nothing to prove. 
Hence we may assume that $|\bar{G}|>2\left|C_{G}(t)\right|^{3}$. By the above consideration, there exists a proper subgroup $\bar{H}$ in $\bar{G}$ such that $|\bar{H}|^{2}>|\bar{G}|$. Hence the full preimage $H$ of $\bar{H}$ in $G$ has the property $|H|^{2}>|G|$. The theorem is proved.

Proof of Theorem 1.2. Recall that the conjugacy class with representative $a$ is called real if $a$ is conjugate with its inverse. If $G$ is a finite group, we denote by $k_{R}(G)$ the number of all real classes of $G$.

We need the following equality, also due to E.P. Wigner (Theorem 1 in [6]):

$$
\sum_{g \in G} \zeta(g)^{2}=k_{R}(/ G)|G|
$$

In particular, for an involution $t$ in $G$ the following holds:

$$
\frac{|G|}{\left|C_{G}(t)\right|^{2}} \leq k_{R}(G) \leq k(G)
$$

Using the class equality, we see that there exists an element $x \in G$ such that $\left|C_{G}(x)\right|>k(G)$. Therefore

$$
\frac{|G|}{\left|C_{G}(t)\right|^{2}} \leq k(G)<\left|C_{G}(x)\right|
$$

If $\left|C_{G}(t)\right|>k(G)$, we have that $|G|<\left|C_{G}(t)\right|^{3}$. If $|G|>\left|C_{G}(t)\right|^{3}$, then $\left|C_{G}(t)\right| \leq$ $k(G)$, and this implies $|G| \leq k(G)^{3}$.

Assume that $|G|>2\left|C_{G}(t)\right|^{3}$ for some involution $t$ in $G$. Then, as in the proof of the first part of Theorem 1, there exists a non-trivial element $x \in G$ such that $\left|G: C_{G}(x)\right| \leq k(G)-1$.

Remark 1. If $G$ is a non-abelian finite simple group, then it follows from (9) that $|G| \leq\left(\left|C_{G}(t)\right|^{2}\right) !$.

Remark 2. Using the classification of finite simple groups it was proved in 4 that there is no non-abelian finite simple group $G$ such that $|G|<k(G)^{3}$, with the exception of $G=L_{2}(q)$ with $q$ even. However, in this case the centralizer of an involution is of order $q$. Therefore for every non-abelian finite simple group $G$ and every involution $t \in G$ we have $|G| \leq\left|C_{G}(t)\right|^{3}$.

\section{REFERENCES}

[1] Brauer R. and Fowler K.A., On groups of even order, Ann. Math. 62 (1955), 565 - 583. MR0074414(17:580e)

[2] Hamermesh M., Group theory and its application to physical problems, Addison-Wesley Publishing Co., Inc., Reading, Mass., London, 1962. MR0136667 (25:132)

[3] Harada K. and Miyamoto M. On the order of a group of even order, J. Algebra 310 (2007), 793 - 800. MR2308180 (2007m:20021)

[4] Kazarin L. and Yanishevsky V., On finite simply reducible groups, St. Petersburg Math. J. 19 (2008), 931 - 951. MR 2411640(2009d:20031)

[5] Lev Arieh, On large subgroups of finite groups, J. Algebra 152 (1992), 434 - 438. MR1194312 (93m:20030) 
[6] Wigner E.P., On representations of certain finite groups, Amer. J. Math. 63 (1941), 57 - 63. MR0003417 (2:216a)

[7] Yamaki H., The order of a group of even order, Proc. Amer. Math. Soc. 136 (2008), 397 - 402. MR2358476 (2008j:20036)

Fachbereich Mathematik, Universität Mainz, D-55099 Mainz, Germany

E-mail address: amberg@mathematik.uni-mainz.de

Department of Mathematics, Yaroslavl State University, 150000 Yaroslavl, Russia

E-mail address: kazarin@uniyar.ac.ru 